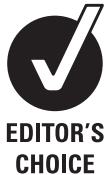

${ }^{1}$ Department of Anesthesiology, Pharmacology \& Therapeutics, University of British Columbia, Vancouver, British Columbia, Canada

${ }^{2}$ Division of Neurosurgery, Department of Surgery, British Columbia Children's Hospital,

Vancouver, Canada

${ }^{3}$ British Columbia Patient Safety and Learning System, Provincial Services Health Authority,

Vancouver, British Columbia, Canada

\section{Correspondence to}

J Mark Ansermino, Department of Anesthesia, British Columbia Children's Hospital, 4480 Oak

Street, Vancouver, British

Columbia, Canada V6H 3V4;

anserminos@yahoo.ca

Accepted 30 March 2009

\title{
Time to listen: a review of methods to solicit patient reports of adverse events
}

\author{
A King, ${ }^{1}$ J Daniels, ${ }^{1}$ J Lim, ${ }^{1}$ D D Cochrane, ${ }^{2}$ A Taylor, ${ }^{3}$ J M Ansermino ${ }^{1}$
}

\section{ABSTRACT}

Background Patients have been shown to report accurate observations of medical errors and adverse events. Various methods of introducing patient reporting into patient safety systems have been published with little consensus among researchers on the most effective method. Terminology for use in patient safety reporting has yet to be standardised.

Methods Two databases, PubMed and MEDLINE, were searched for literature on patient reporting of medical errors and adverse events. Comparisons were performed to identify the optimal method for eliciting patient initiated events.

Results Seventeen journal publications were reviewed by patient population, type of healthcare setting, contact method, reporting method, duration, terminology and reported response rate.

Conclusion Few patient reporting studies have been published, and those identified in this review covered a wide range of methods in diverse settings. Definitive comparisons and conclusions are not possible. Patient reporting has been shown to be reliable. Higher incident rates were observed when open-ended questions were used and when respondents were asked about personal experiences in hospital and primary care. Future patient reporting systems will need a balance of closed-ended questions for cause analysis and classification, and openended narratives to allow for patient's limited understanding of terminology. Establishing the method of reporting that is most efficient in collecting reliable reports and standardising terminology for patient use should be the focus of future research.

Adverse events represent a significant challenge in effective healthcare provision worldwide. An adverse event is defined as an injury resulting from medical management rather than from an underlying illness. ${ }^{1}$ Accurate identification and reporting of adverse events is needed to enable learning and prevent recurrence.

Traditional adverse event reporting systems rely on the healthcare provider to report events. The World Health Organization has developed guidelines and terminology for use in healthcare provider reporting systems. ${ }^{2}$ However, it is patients who are most affected by adverse events. Involving the patient in reporting provides a direct benefit to those affected and captures details of events not available through other reporting techniques. ${ }^{3}$ As patient reporting is a relatively new addition to patient safety reporting systems, the techniques that are most successful and efficient are not yet known. Standardised guidelines on methods and terminology have yet to be developed. This litera- ture review attempts to identify the state of the art in patient reporting systems used in research studies and reviews the healthcare setting populations, contact methods, verification, reporting methods, incentives, incident rates and terminology used for patient reports of adverse events. Successful approaches used in research studies may have wider application to general hospital or outpatient clinic operations, particularly in quality and safety improvement initiatives, providing they can be made acceptable to users. ${ }^{45}$

\section{METHODS}

A search for relevant literature was carried out using the MEDLINE OvidSP (1950 to present) and PubMed (1949 to present) databases between January and April 2008. These searches were conducted with a combination of keywords relating to patient safety and patient reporting. We searched Pubmed for the terms Patient (MeSH term) survey ("data collection" (MeSH term)) AND medical events; Patient (MeSH term) perception (MeSH term) AND adverse events; patient (MeSH term) perception (MeSH termterm) AND "medical error" (MeSH term); Patient (MeSH term) survey ("data collection"(MeSH term)) and quality improvement; Patient (MeSH term) reported AND medical errors (MeSH term); "Patient survey" AND 'safety'. In addition, we searched MEDLINE for the terms Safety (MeSH term) AND adverse event reporting; patient (MeSH term) reported AND medical error (MeSH term) or undesirable events; Patient (MeSH term) reports AND safety (MeSH term) AND adverse events; Error reporting patients (MeSH term). The searches were limited to publications in English. Searches returning $>200$ papers were further filtered by additional keywords. Reference lists were used to locate additional papers.

After the search was conducted (see figure 1 for search strategy), the abstracts of the resulting publications were examined to determine their applicability. Relevant publications were defined to be those that collected reports from patients about medical errors or adverse events experienced in healthcare. Titles and abstracts were reviewed by one of the authors. Two other further authors independently confirmed the eligibility with full manuscript review. Reports of malpractice litigation and closed claims studies were excluded. All other discovered forms of safety or quality defect reporting from patients, such as spontaneous complaints, satisfaction surveys, research studies and systems designed for patient input were within the scope of the review. Papers meeting these inclusion criteria were further evaluated based on 
healthcare setting population, contact method, incentives for report completion, reporting methods, reporting terminology, methods for corroborating patient reports and reporting types and rates.

\section{RESULTS}

Ninety combination keyword database searches identified 11 relevant publications, two of which used the same data sets, and two papers were located from reference lists (see figure 1). Of the publications that were rejected after screening, three were found to be focused on the patient's perception of medical errors, two detailed quality of care issues and one pertained to error prevention involving patients. Four additional papers were suggested by colleagues, bringing the total to 17 .

\section{Healthcare setting}

The healthcare settings surveyed in these papers varied widely. Five papers asked patients about mistakes encountered involving any aspect of healthcare, including emergency and ambulatory care, and six papers asked about errors during hospitalisation. Four papers focused on errors in primary healthcare, and an additional paper's scope included both primary and specialty care. The remaining study surveyed oncology patients in a teaching hospital.

\section{Solicitation and study duration}

Study participants were either involved via self-initiated interest or actively solicited. Eleven papers (65\%) elicited patient reports, whereas the remaining five used self-initiated reporting surveys. ${ }^{3}{ }^{6-21}$ On average, more reports were collected from solicited patients than self-initiated participants. The shortest time period of study was 5 days, whereas the longest was 2 years. ${ }^{12} 16$ Approximately a third (35\%) of the studies collected reports over periods ranging from 2 to 4 months. ${ }^{3} 67101114$

\section{Incentives}

Two studies used incentives to encourage reporting. ${ }^{8}{ }^{16} \mathrm{~A}$ recruitment technique involving random telephone number dialing and offering a $\$ 50$ payment for an in-person interview yielded one study participant per 10 to 20 calls. Thirty-eight usable interviews resulted from this recruitment method. ${ }^{8}$ The study with the largest number of patient responses used an online survey with customised health and self-management resources as an incentive for participation. ${ }^{16}$

\section{Reporting methods and response rate}

The methods used for collecting patient reports varied along with response rates (see table 1 for the terms used when asking patients about adverse events). Recruitment by random digit dialing was not used in any of the hospital patient studies; however, this method achieved the highest response rate of the five studies focused on broad healthcare experiences. ${ }^{6}$ Primary care patient reporting studies used a combination of methods: one used telephone recruitment with a follow up in-person interview; another allowed patients to choose written, online or telephone reporting; and a third used telephone survey. ${ }^{8} 1221$ Interviewing patients in-person was effective in obtaining high response rates from hospital patients (average 87\%) compared to telephone reports from non-hospital settings (average 44\%). The highest response rate overall was $96 \%$, achieved by in-person patient advocate interviews for a specific hospital unit. ${ }^{15}$ The study with the highest number of responses, over a 2-year period, was a reporting system for various healthcare setting experiences with 44860 responses. ${ }^{16}$

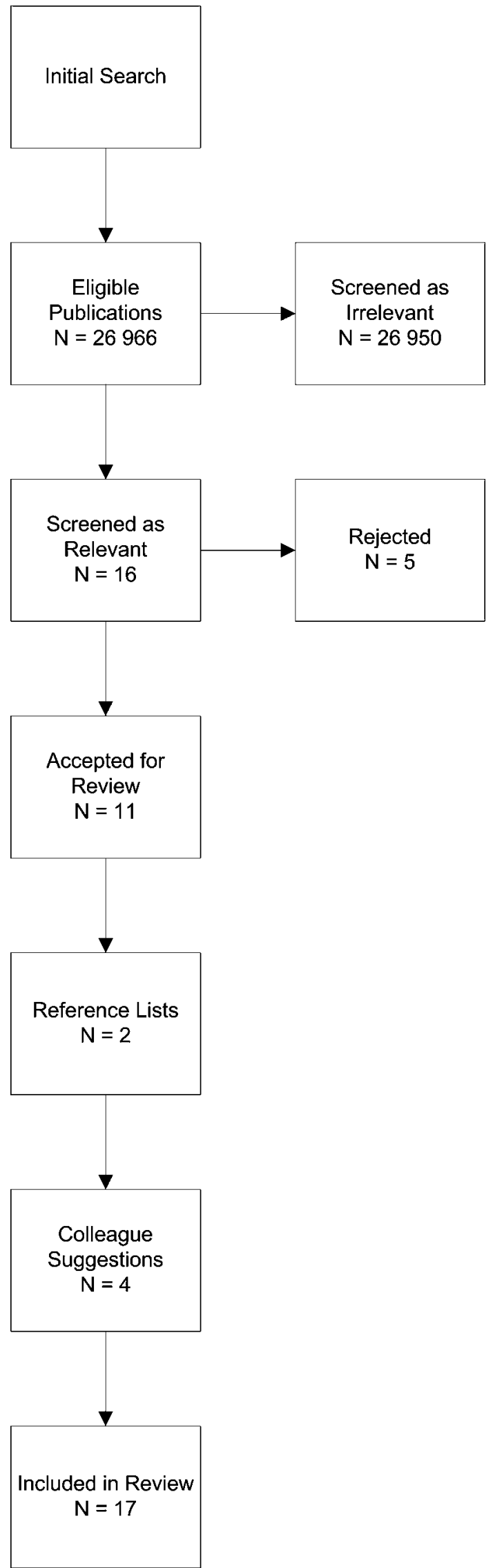

Figure 1 Schematic of literature search strategy. 
Table 1 Terminology for patient reporting of adverse events

Term used
Medical error, mistake ${ }^{3610141618}$
Medical mistake
Comments (stand-alone kiosk in hospital)
Preventable incidents $^{8}$
Unsafe $^{15}$
Complications, problems, negative effects, or unexpected or unpleasant situations ${ }^{919}$
Safety related undesirable events ${ }^{17}$
Problem or injury ${ }^{32}$
Anything ever go wrong
Should not have happened and that you don't want to see happen again
Symptomatic inquiry

\section{Corroboration}

Patient reports of adverse events were corroborated in three $(18 \%)$ publications. One study reviewed medical records, whereas the other two compared patient reported incidents to hospital incident reports and/or incidence rates reported in the literature. All studies that performed corroboration targeted hospitalised patients. ${ }^{3} 911$ Cross-referencing medical charts, physician notes and orders, and nurse notes proved to be an effective method for inpatients. ${ }^{3}$ The incidence of nosocomial infections, pressure ulcers and drug-related events reported by patients was shown to be comparable to rates documented by healthcare providers in hospital and to rates reported in the patient safety literature. ${ }^{9}$

\section{Report characteristics}

The incidence rate for adverse events across settings and populations varied considerably, ranging from less than 0.1 to 5.8 per patient. $^{8} 1620$ Incident rates in the target populations and healthcare settings varied widely and thus were not statistically comparable. More than half (55\%) of studies targeting hospitals or primary care settings reported a rate of one incident or more per person, whereas surveys covering a broad range of healthcare environments reported a rate of 0.6 incidents or fewer per person. Disregarding any other differences in reporting method, five studies used only open-ended questions, averaging 1.9 reported incidents per person, whereas strictly closed-ended questions or a combination of both types achieved averages of 0.7 and 0.4 per person, respectively. Incident rates from reports of personal experiences averaged 1.3 per person, whereas rates from reporters including family or household members' experiences averaged 0.3 incidents per person.

Classification of reports was inconsistent among publications. Eight studies (47\%) used reporter self-assessment, five had clinicians review reports, three authorised researchers to classify cate- gories and one had lawyers evaluate possible compensation. ${ }^{36-20}$ Severity of health consequences was used to classify events in five studies. ${ }^{3} 6111516$ Table 2 shows the relationship between study setting and reporting method.

\section{DISCUSSION}

The publications reviewed in this paper varied considerably in terms of healthcare setting, method of reporting, time span, terminology, criteria for assessment and response rate. Openended questions, and solicitation techniques based exclusively on personal experiences tended to yield higher incident rates. $^{3}$ 7-9 12131517 Patient reporting within a specific hospital unit using in-person patient advocate interviews had the highest response rate. ${ }^{15}$ With only two studies using incentives, and with each using a different incentive, there is insufficient evidence to conclude whether incentives increase response rate. ${ }^{8} 16$ There have been too few studies for definitive conclusions on which terminology could be most effectively used with patient reporting. Recall bias has been identified as a limitation in patient reporting. ${ }^{911}$ At the present time, there is marginal evidence indicating that in-person and open-ended interview techniques are preferable to non-personally mediated closed-ended interview techniques. Future policy research is needed to determine the optimal use of language and setting for patient reporting.

\section{Reporting}

Healthcare setting

The 13 publications in this review ranged in setting, focusing on specific wards, hospitals, primary care facilities, or a combination thereof (table 3 ). The types and frequency of errors and adverse events in each are idiosyncratic, limiting generalisability. However, reporting within a hospital setting has been associated with higher response rates. 3891117

\section{Accuracy of patient reports}

Whenever investigated, patients have been shown to be capable of reporting medical errors accurately. ${ }^{3} 911$ Ensuring that adverse events are documented soon after occurrence would decrease recall bias for healthcare providers and patients, and would parallel a novel and promising new approach for detecting nonroutine events during anaesthesia. ${ }^{22}$

\section{Reporting structure}

Recruiting and interviewing in-person within hospital and primary care settings tended to increase response rates. It has been demonstrated that patients can effectively use online surveys, which are both easily accessible and cost-effective. ${ }^{16} 20$ Exclusively asking for personal experiences or using open-ended questions may yield higher incident rates but requires more time

Table 2 Relationship between study setting and reporting method

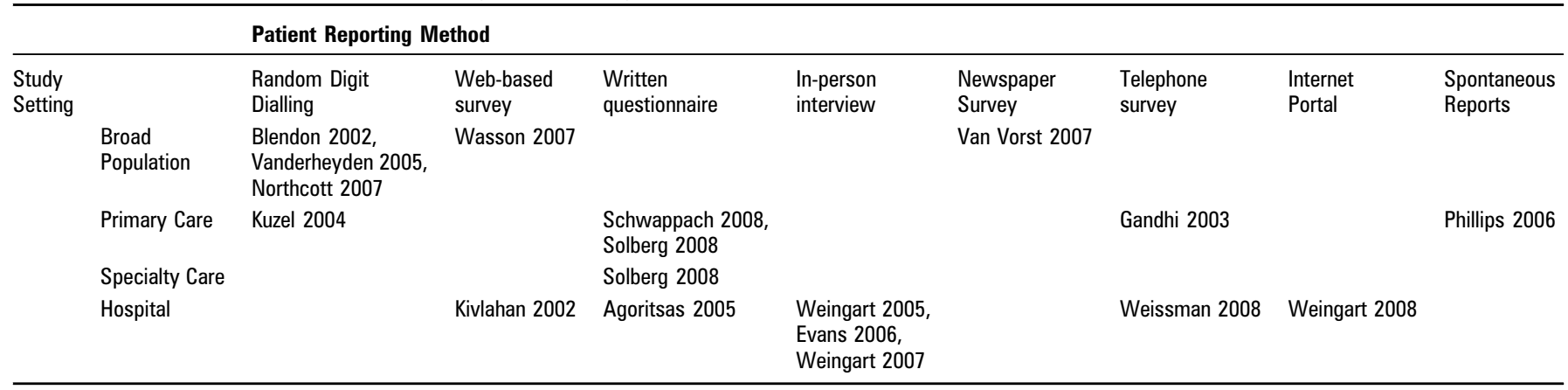




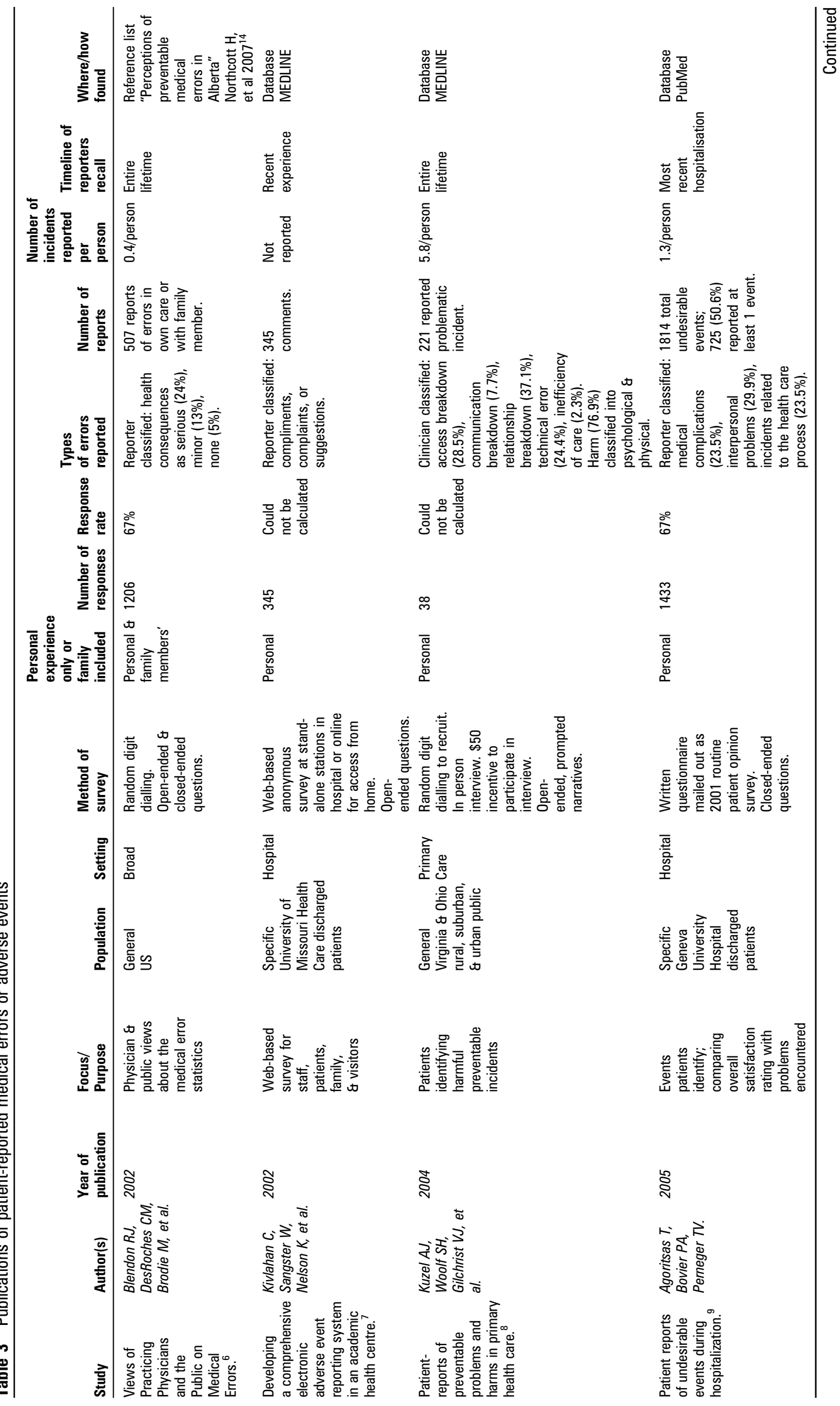




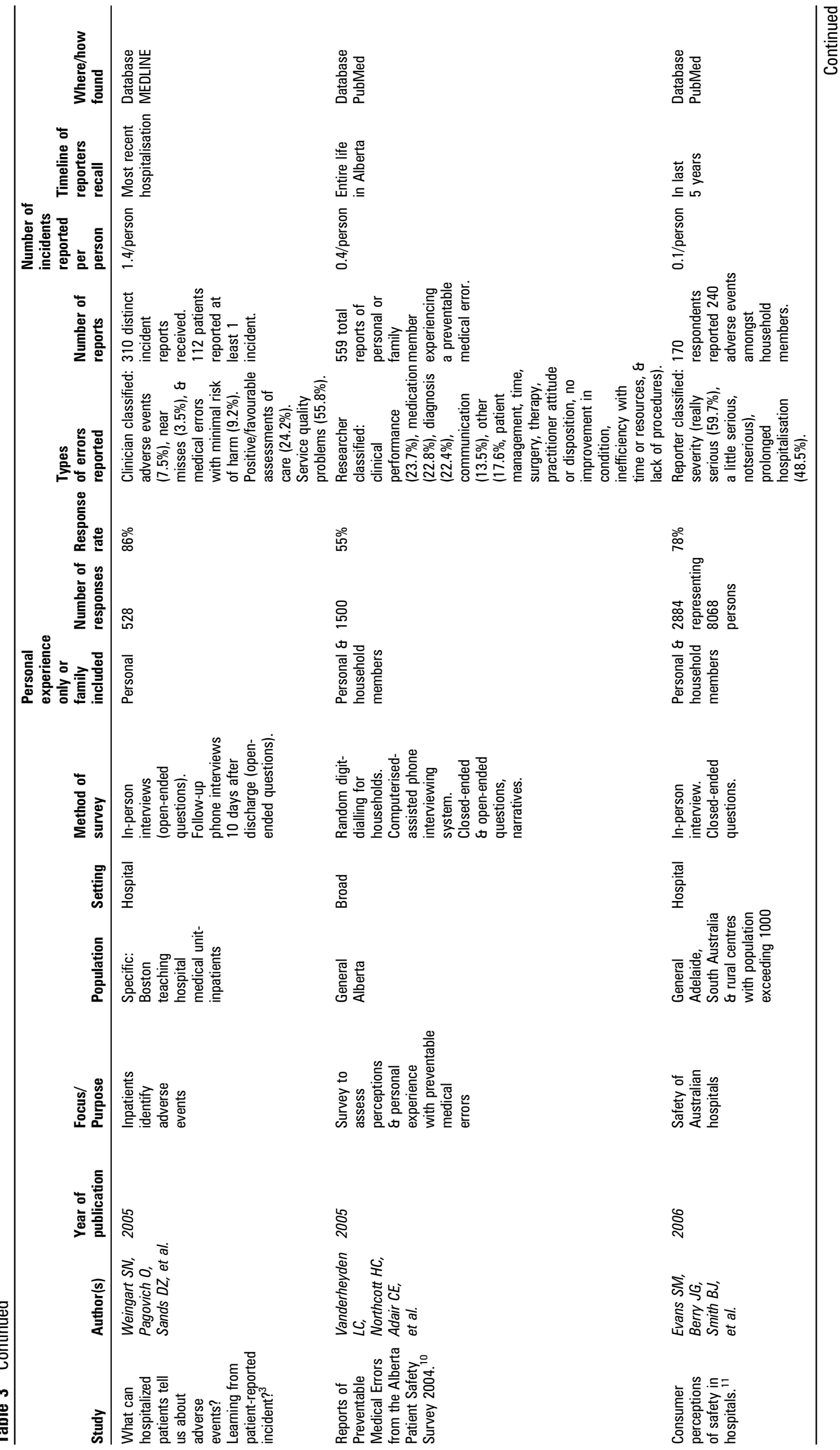




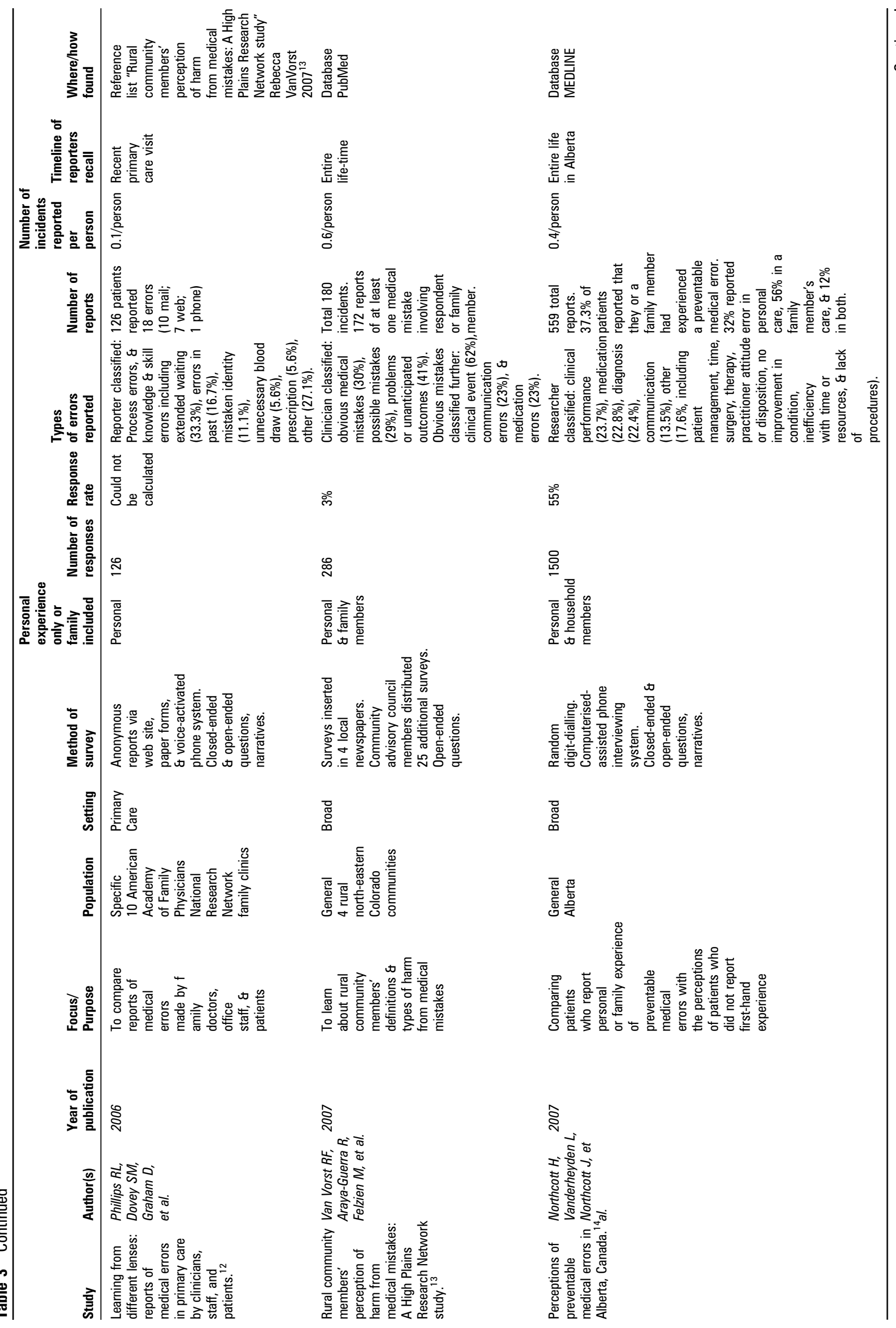



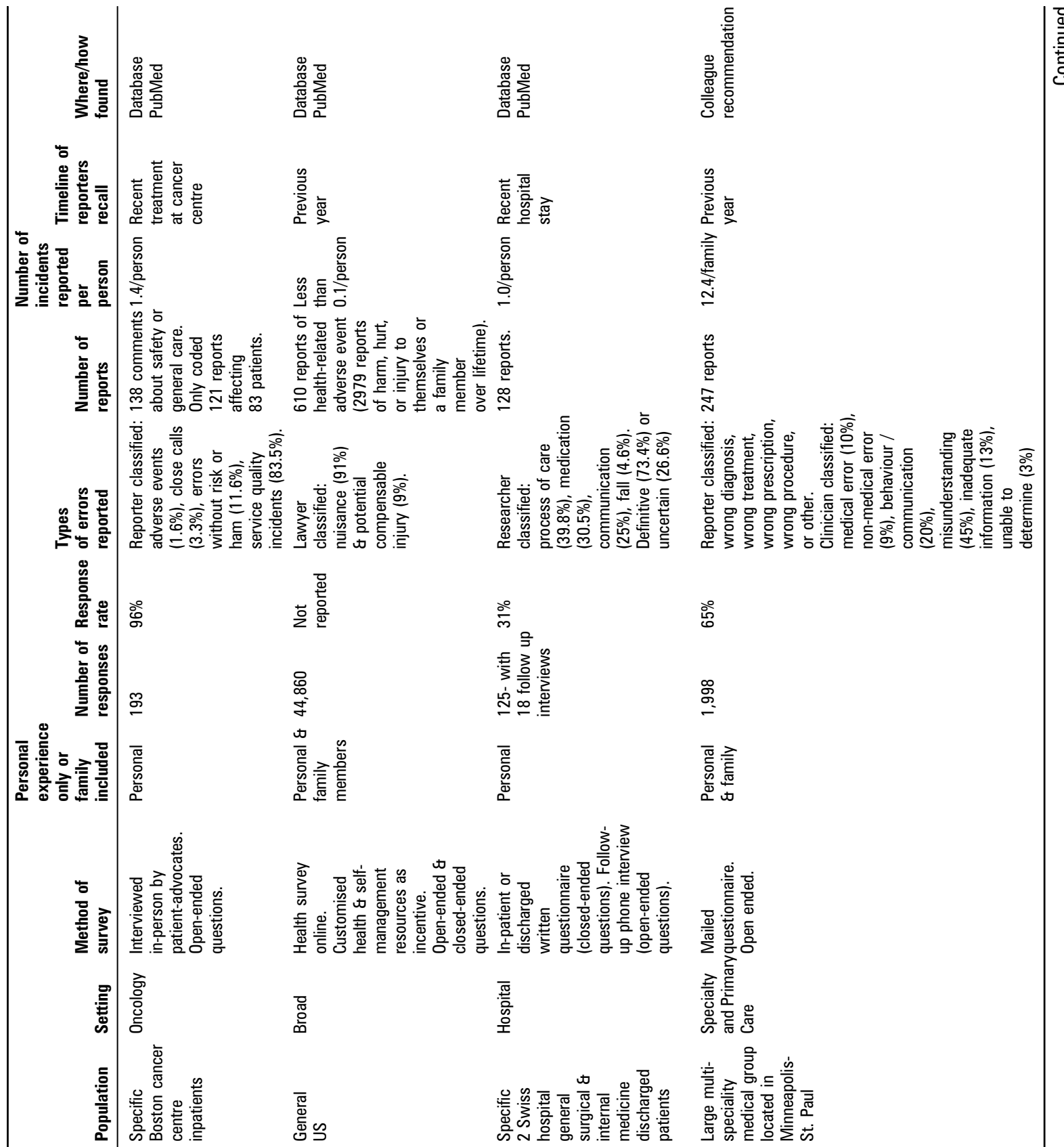

$\stackrel{\circ}{\text { ڤ }}$

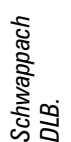

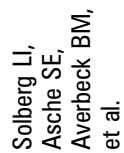




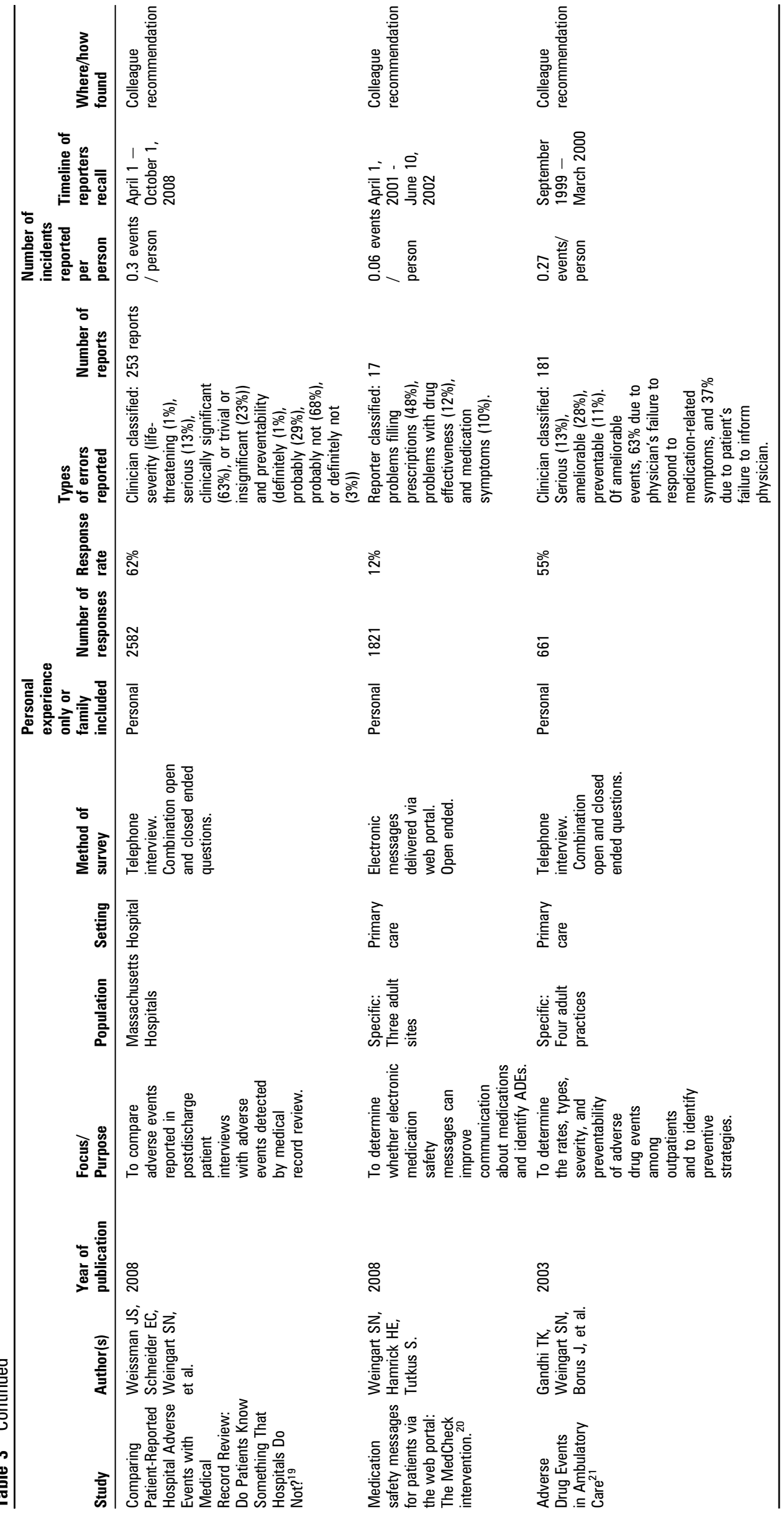


for analysis. The trends observed in response rate are not statistically significant and could be due to numerous factors, such as population sample size or terminology and assessment schemes used for classifying a patient-reported adverse event. Therefore, these comparisons are limited in providing recommendations for future reporting systems.

A combination of closed-ended questions and open-ended narratives may be the most effective for soliciting reports and data for analysis. Direct questions and limited response options allow for accurate analysis and provide a structure for classifying adverse events and near misses but do not allow patients to explain details of events. ${ }^{3} 91617$ Patients' fear of providing inaccurate observations was alleviated by refining survey options to allow for reporting a "possible" event. ${ }^{17}$ An alternative method initially provides a definition of adverse event, then allows respondents to narrate experiences; analysis is based on a standardised patient safety definition. 3810121415

\section{Terminology}

The language used to solicit responses from patients and families about adverse events or near misses can have a significant impact on what is reported. Reports solicited from patients are likely to increase when lay language is used. Patients may prefer the term "mistake" to "medical error". ${ }^{6}$ Numerous alternative terms have been used for soliciting reports from patients, as seen in table 3 , with little consensus on which term is the most reliable for patient reports.

Common terms used by patient safety professionals, such as adverse event or medical error, are often misunderstood by the general public. ${ }^{10} 1323$ Patient reporting literature suggests that the use of lay language is more effective in soliciting reports. To facilitate patient reporting, different terminology is required and will need to be developed.

\begin{abstract}
Limitations
The search strategy, including the search terms used, is a limitation of this research. Relevant publications that were not found because of the use of only the two databases could bias our findings. This review is based on a small sample size in very diverse settings. Consensus among so few studies that vary in focus and methods is unattainable and does not permit statistical analysis.
\end{abstract}

\section{Recommendations}

Further research is required to identify the optimal language, method of report solicitation, reporting tool and incentive in each specific clinical setting. By using the patient as a source of input to a health system, an obligation is established that requires the system to respond to concerns and address issues raised. How to close the loop with the reporter, especially if the reporting is anonymous, requires further definition. A sustained cycle of event identification and quality improvement should be the goal of future efforts to translate this research into clinical practice. Unlike many of the studies identified, future studies should be conducted as a sustainable process within the clinical environment. A patient reporting system should support a learning and action system and be an integral part of every clinical environment.

Potential issues affecting confidentiality remain to be elucidated and resolved. Improving the reporting of adverse events especially when patient advocates are actively involved in soliciting these reports may compromise confidentiality for patients or staff members. Research will be required to identify and mitigate these concerns and harness the advantages of patient advocates.
Based on the studies reviewed, a number of recommendations can be made for the design and implementation of future reporting systems. When designing a reporting tool, it should be evaluated in the local setting to ensure appropriate terminology is used. International terminology standards should be adopted, or translation tables developed, to ensure general applicability of results. Reports from patients should be actively solicited. This work should ideally be undertaken in person as soon after the event as possible; however, telephone interviews do produce acceptable response rates. Incentives increase response rates and should be considered. Efforts should be made to corroborate patient reports.

From the policy perspective, the engagement of patients in reporting for the purposes of learning from their experiences changes the obligations a health system or institution has to the patient. Although engaging patients strengthens the patientcentred focus of an organisation, it also requires that actions are taken and improvements made on issues identified in the reports. Patients have a personal interest in seeing improvements made and risks mitigated. In the future, patients will become a key component of implementing quality improvement initiatives.

\section{CONCLUSIONS}

Families and patients, rather than healthcare providers exclusively, can be involved with improving safety in healthcare. As the patient is the one true constant in care, actively and consistently collecting observations about the healthcare experience provides a valuable perspective for improving patient safety. The reliability of patient reporting of adverse events has been established as trustworthy, and using these reports as part of patient safety learning systems could identify problems that currently go unreported in healthcare provider reporting systems. ${ }^{3} 911$ The most efficient method for each healthcare setting and the best terminology to use with patients for collecting adverse events reports are still unknown. Taking a lead from anaesthesia safety research, reporting immediately after an incident could address recall bias among reporters, both healthcare providers and patients. ${ }^{22}$ There have been too few studies for definitive conclusions and the studies that have been conducted are too diverse to compare statistically. On a positive note, the World Health Organization's work on an International Classification for Patient Safety is helping to standardise the definitions used for adverse event reporting, which will aid in attempts to compare different reporting systems.

The overall objective for reporting systems must stay in focus. Patient reporting systems could enhance patient safety by increasing follow-up by healthcare providers, analysis of trends, identification of causes and, most importantly, implementation of solutions. Reporting on its own is insufficient to increase patient safety.

Funding This study was funded in part by grant \#PSI 85002 from the Canadian Patient Safety Institute administered by the Canadian Institutes of Health Research. Funding sources had no involvement in study design, analysis or dissemination of results.

Competing interests None.

Patient consent Obtained.

Provenance and peer review Not commissioned; externally peer reviewed.

\section{REFERENCES}

1. Massachusetts Coalition for the Prevention of Medical Errors (MCPME). When things go wrong: responding to adverse events. Institute for Healthcare Improvement, 2006. http://www.ihi.org/NR/rdonlyres/A4CE6C77-F65C-4F34-B32320AA4E41DC79/0/RespondingAdverseEvents.pdf (accessed 7 Aug 2008). 
2. World Health Organisation (WHO). WHO draft guidelines for adverse event reporting and learning systems. Geneva, Switzerland: WHO Press, 2005. http://www. who.int/patientsafety/events/05/Reporting Guidelines.pdf (accessed 7 Aug 2008).

3. Weingart SN, Pagovich 0 , Sands DZ, et al. What can hospitalised patients tell us about adverse events? Learning from patient-reported incidents. J Gen Intern Med 2005;20:830-6.

4. Pronovost PJ, Berenholtz SM, Goeschel C, et al. Improving patient safety in intensive care units in Michigan. J Crit Care 2008;23:207-21.

5. Gaba DM. Anaesthesiology as a model for patient safety in health care. Brit Med Assoc J. 2000;320:785-8.

6. Blendon RJ, DesRoches $\mathrm{CM}$, Brodie $\mathrm{M}$, et al. Views of practicing physicians and the public on medical errors. N Engl J Med 2002;347:1933-40.

7. Kivlahan C, Sangster W, Nelson K, et al. Developing a comprehensive electronic adverse event reporting system in an academic health center. Jt Comm J Qual Improv 2002:23:583-94.

8. Kuzel AJ, Woolf SH, Gilchrist VJ, et al. Patient reports of preventable problems and harms in primary health care. Ann Med Fam 2004;2:333-40.

9. Agoritsas T, Bovier PA, Perneger TV. Patient reports of undesirable events during hospitalisation. J Gen Intern Med 2005;20:922-8.

10. Vanderheyden LC, Northcott HC, Adair CE, et al. Reports of preventable medical errors from the Alberta patient safety survey 2004. Healthc 0 2005:8:107-14

11. Evans SM, Berry JG, Smith BJ, et al. Consumer perceptions of safety in hospitals BMC Public Health 2006;6:41-7.

12. Phillips RL, Dovey SM, Graham D, et al. Learning from different lenses: reports of medical errors in primary care by clinicians, staff and patients. J Patient Saf 2006;2:140-6
13. Van Vorst RF, Araya-Guerra R, Felzien M, et al. Rural community members' perceptions of harm from medical mistakes: a High Plains Research Network (HPRN) study. J Am Board Fam Med 2007;20:135-43.

14. Northcott H, Vanderheyden L, Northcott J, et al. Perceptions of preventable medical errors in Alberta, Canada. Int $J$ Qual Health Care 2008;20:115-22.

15. Weingart SN, Price J, Duncombe D, et al. Patient-reported safety and quality of care in outpatient oncology. J Qual Patient Saf 2007;33:83-94.

16. Wasson JH, MacKenzie TA, Hall M. Patients use an internet technology to report when things go wrong. Qual Saf Health Care 2007:16:213-15.

17. Schwappach DLB. "Against the silence": development and first results of a patient survey to assess experiences of safety-related events in hospital. BMC Health Services Research 2008:8:59-66.

18. Solberg LI, Asche SE, Averbeck BM, et al. Can patient safety be measured by surveys of patient experiences? Jt Comm J Qual Patient Saf 2008:34:266-74

19. Weissman JS, Schneider EC, Weingart SN, et al. Do patients know something that hospitals do not? Ann Intern Med 2008;149:100-8.

20. Weingart SN, Hamrick HE, Tutkus $S$, et al. Medication safety messages for patients via the Web portal: the MedCheck intervention. Int J Med Inform 2008; 77:161-8.

21. Gandhi TK, Weingart SN, Borus J, et al. Adverse drug events in ambulatory care. N Engl J Med 2003;348:1566-4.

22. Oken A, Rasmussen MD, Slagle JM, et al. A facilitated survey instrument captures significantly more anesthesia events than does traditional voluntary event reporting. Anesthesiology 2007;107:909-22.

23. Burroughs TE, Waterman AD, Gallagher TH, et al. Patients' concerns about medical errors during hospitalization. J Qual Patient Saf 2007;33:5-14. 